\title{
Desigualdade e polarização entre empregados na agricultura brasileira: 1992-2007
}

\section{Rodolfo Hoffmann ${ }^{1}$}

\section{Resumo}

Após discutir o conceito de polarização de uma distribuição de renda, é analisada a distribuição do rendimento do trabalho principal dos empregados na agricultura brasileira no período 1992-2007. Considerando o período 1995-2007, após a estabilização monetária, verifica-se que a desigualdade daquela distribuição é estável e que o grau de polarização apresenta tendência decrescente. Também é examinada a evolução da desigualdade entre empregados permanentes e temporários e entre empregados sem carteira e com carteira de trabalho assinada, destacando o diferente papel desempenhado pelo salário mínimo na distribuição da renda dentro dessas categorias de trabalhadores. Finalmente, mostra-se que houve crescimento da participação do componente interregional na desigualdade da distribuição dos salários na agricultura brasileira.

Palavras-chave: Polarização, Desigualdade, Distribuição de renda, Agricultura - Brasil.

\begin{abstract}
The paper discusses the concept of polarization of an income distribution and examines the earnings distribution of employees in Brazilian agriculture in the period 1992-2007. Considering the period 1995-2007, after the monetary stabilization, it is shown that the inequality of that distribution is stable and that its degree of polarization has a decreasing trend. Next, the paper analyses the changes in the inequality between permanent and temporary employees and between those with or without the legal employee's document, pointing to the different role of the legal minimum wage in influencing the earnings distribution within these worker categories. Finally, the paper shows that the share of regional differences in the inequality of the distribution of wages in Brazilian agriculture has increased.
\end{abstract}

Key words: Polarization, Inequality, Income distribution, Agriculture - Brazil.

JEL D31, D39, J43.

\section{Introdução}

Artigo de Balsadi e Graziano da Silva (2008) analisa "a polarização da qualidade do emprego na agricultura brasileira no período 1992-2004”.2 O artigo evidencia a existência de discrepâncias na qualidade do emprego agrícola entre diferentes categorias de empregados, mas não apresenta nenhuma medida específica de polarização ou de desigualdade. Consequentemente, não fica esclarecido se há ou não uma tendência de aumento de polarização no mercado de trabalho agrícola no país.

(1) Professor do Instituto de Economia da Universidade Estadual de Campinas (IE/Unicamp), com apoio do CNPq (Conselho Nacional de Desenvolvimento Científico e Tecnológico), Campinas, SP, Brasil.

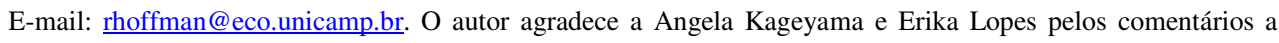
uma versão preliminar do texto.

(2) A mesma análise é apresentada no capítulo 4 do livro de Balsadi (2008). 
Rodolfo Hoffmann

Pretende-se, neste texto, discutir melhor o conceito de "polarização" e, em seguida, aprofundar e atualizar parte da análise estatística desenvolvida por Balsadi e Graziano da Silva (2008).

\section{Polarização}

Para que o uso do termo "polarização" contribua para aprofundar a análise de fenômenos socioeconômicos, é necessário que lhe seja atribuído um sentido específico, distinto do conceito de desigualdade ou da simples existência de diferenças. O uso de "polarização" como sinônimo de "desigualdade" não representa nenhum aprofundamento da análise científica.

Dispomos de inúmeras medidas da desigualdade de uma distribuição, cabendo destacar o índice de Gini e as medidas $T$ e $L$ de Theil.

Um trabalho pioneiro no que se refere à conceituação e mensuração da polarização da distribuição da renda foi feito por Wolfson (1994). A mediana delimita a metade mais pobre e a metade mais rica da população. Seja $G_{e}$ o índice de Gini da desigualdade entre esses dois grupos e seja $G$ o índice de Gini de toda a distribuição. Sendo $\mu$ a renda média e $n$ a renda mediana, a medida de polarização de Wolfson é

$$
P_{W}=2\left(2 G_{e}-G\right) \frac{\mu}{m}
$$

Note-se que a desigualdade dentro dos dois grupos corresponde a $G-G_{e}$. Então a diferença entre a desigualdade entre os grupos e a parcela referente à desigualdade dentro dos grupos é

$$
G_{e}-\left(G-G_{e}\right)=2 G_{e}-G
$$

Verifica-se que essa expressão constitui o núcleo da medida de polarização de Wolfson [expressão (1)].

Se a média $(\mu)$ for adotada como limite entre os relativamente pobres e os relativamente ricos, e o índice de Gini da desigualdade entre esses grupos for indicado por $G_{M}$, obtemos, analogamente, uma outra medida de polarização:

$$
P_{M}=2\left(2 G_{M}-G\right)
$$

Mudando a maneira de delimitar os relativamente pobres e os relativamente ricos e variando a medida de desigualdade adotada, é claro que podem ser definidas inúmeras medidas de polarização, sempre respeitando o conceito de que maior polarização significa uma maior desigualdade entre esses grupos, em comparação com a medida da desigualdade dentro dos grupos ou com a medida geral de desigualdade. Um exemplo numérico deve ajudar a esclarecer o conceito de polarização, como algo associado, mas distinto da desigualdade. A Tabela 1 mostra duas maneiras de distribuir a mesma renda total entre 4 pessoas. 
Tabela 1

Duas maneiras ( $\alpha$ e $\beta$ ) de distribuir a mesma renda total entre 4 pessoas

\begin{tabular}{c|c|c}
\hline Pessoa & Renda $(\alpha)$ & Renda $(\beta)$ \\
\hline A & 0 & 2 \\
\hline B & 4 & 2 \\
\hline C & 4 & 6 \\
\hline D & 8 & 6 \\
\hline Total & 16 & 16 \\
\hline
\end{tabular}

Nas duas distribuições a renda média e a renda mediana são iguais a 4. Uma vez que é possível passar da distribuição $\alpha$ para a distribuição $\beta$ fazendo duas transferências progressivas (transferindo 2 de B para A e 2 de D para C), a desigualdade é menor na distribuição $\beta$. O índice de Gini é igual a 0,375 na distribuição $\alpha$ e 0,250 na distribuição $\beta$. Mas a polarização é maior na distribuição $\beta$. Na distribuição $\alpha$ há uma "classe média" de duas pessoas cuja renda é igual à média ou mediana, o que não ocorre na distribuição $\beta$. Para a distribuição $\alpha$ tem-se $P_{W}=P_{M}=0,25$ e para a distribuição $\beta$ verifica-se que $P_{W}=P_{M}=0,50$.

O conceito analisado é o de bipolarização. Não será abordada aqui a medida mais geral proposta por Esteban e Ray (1994), que permite considerar mais de dois grupos. ${ }^{3}$

Se a divisão da população em grupos for feita com base em algum critério que não seja o de estratos de renda, o índice de Gini global não é composto apenas pelo índice de Gini entre grupos e uma parcela referente à desigualdade dentro deles, existindo ainda uma parcela associada com a superposição das distribuições de renda dos grupos. ${ }^{4}$ Isso invalida o raciocínio seguido para chegar às medidas de polarização (1) e (3). Uma alternativa é considerar a participação percentual da medida de desigualdade entre grupos na desigualdade global. De qualquer maneira, se a variável utilizada para delimitar os grupos não é a renda, já não se trata, stricto sensu, do conceito de polarização da distribuição da renda.

\section{Os dados}

Para analisar a desigualdade e a polarização entre empregados na agricultura brasileira de 1992 a 2007, serão utilizados os microdados da PNAD (Pesquisa Nacional por Amostra de Domicílios) para esse período.

Conforme o procedimento adotado por Balsadi e Graziano (2008), não se considera a área rural da região Norte (inclusive Tocantins) e são incluídas apenas as pessoas empregadas no setor agrícola (variável da PNAD V4709 ou V4809 igual a 1), com ou sem carteira de trabalho assinada (variável V4706 igual a 1 ou 4, respectivamente), classificada como empregado temporário (V9008 igual a 4) ou empregado permanente na agricultura, silvicultura ou criação de bovinos, bubalinos, caprinos, ovinos ou suínos (V9008 igual a 2).

(3) Referências adicionais podem ser encontradas em Hoffmann (2008).

(4) Ver, por exemplo, Hoffmann (1998, seção 3.10). 
Considera-se o rendimento mensal do trabalho principal, expresso em reais de setembro-outubro de 2007, com base no INPC. Dada a importância dessa variável na análise e tendo em vista o uso da medida de desigualdade $L$ de Theil (que não é definida quando há rendas nulas), optou-se por excluir as observações com rendimento do trabalho principal não declarado ou igual a zero.

Conforme dados da PNAD de 2007, para o conjunto de pessoas analisadas com informação do valor do rendimento de todas as fontes, o salário recebido na atividade principal representa 98,6\% do rendimento de todos os trabalhos e 93,2\% do rendimento de todas as fontes. Ao analisar os resultados, é necessário ter em mente as limitações dos dados sobre renda na PNAD, que tendem a ser subdeclarados. Particularmente no caso da agricultura, cabe lembrar que os rendimentos informados não incluem o valor da produção para autoconsumo. No caso de um trabalhador temporário, é possível que o rendimento mensal no mês de referência da pesquisa seja uma superestimação do seu rendimento médio no ano, se ele fica um período desempregado.

Todas as análises estatísticas são ponderadas, utilizando a última versão dos fatores de expansão fornecidos pelo IBGE.

Para calcular a escolaridade média, adotou-se um valor de 17 para as pessoas com 15 anos ou mais de escolaridade. No cálculo da escolaridade média foi necessário, obviamente, excluir as observações sem declaração de escolaridade. Mas essas observações não foram excluídas no caso de análises que não envolviam a escolaridade. Dessa maneira, o número de observações utilizado no cálculo da escolaridade média (8.025 em 2007) é um pouco inferior ao número de observações utilizado no cálculo das medidas de desigualdade da distribuição da renda (8.044 em 2007).

Os resultados serão apresentados para o período 1992-2007, mas optou-se por destacar as mudanças nas medidas de desigualdade e polarização durante o período 1995 2007, tendo em vista os efeitos da instabilidade inflacionária sobre os resultados referentes a 1992 e 1993.

Para verificar a existência de tendência crescente ou decrescente dos diferentes indicadores no período 1995-2007, foi ajustada uma regressão linear simples do indicador contra o tempo (em anos), com 12 pontos observados, e foi testada a hipótese de nulidade do coeficiente angular.

\section{Variação da desigualdade e da polarização da distribuição da renda}

A Tabela 2 apresenta algumas informações básicas sobre a população analisada. O número de empregados agrícolas no país (excluindo sempre a área rural da região Norte) cai substancialmente de 1992 a 1998 e depois mostra tendência crescente até 2005. Nos últimos dois anos do período analisado essa população cai de 4,5 para 4,3 milhões de pessoas.

A idade média desses empregados cresce ao longo de todo o período 1992-2007.

O rendimento real médio do trabalho principal cresce substancialmente entre 1993 e 1996 e depois diminui até 2003. De 2003 a 2007 há crescimento intenso (mais de 7\% por ano), acompanhando o crescimento do valor real do salário mínimo.

Desde 1995 o rendimento mediano coincide ou está muito próximo do salário mínimo, cujo valor real cresce 67\% de setembro de 1995 a setembro de 2007. Cabe 
ressaltar que é elevada a frequiência de rendimentos iguais ao salário mínimo. Em 2007, todos os percentis do $45^{\circ}$ ao $60^{\circ}$ são iguais ao salário mínimo, indicando que pelo menos $15 \%$ dos valores declarados são iguais a esse limite legal. ${ }^{5}$

Tabela 2

Número de empregados agrícolas, sua idade média, valor médio e mediano do rendimento da atividade principal e valor real do salário mínimo em setembro-outubro de cada ano. Brasil,

1992-2007

\begin{tabular}{c|c|c|c|c|c}
\hline \multirow{2}{*}{ Ano } & \multirow{2}{*}{ No $(1000)$} & \multirow{2}{*}{ Idade Média } & \multicolumn{2}{|c|}{ Rendimento } & \multirow{2}{*}{$\begin{array}{c}\text { Salário } \\
\text { Nínimo real }\end{array}$} \\
\cline { 4 - 5 } & & & Médio & Mediano & Mín \\
\hline 1992 & 4769 & 32,5 & 269,8 & 219,5 & 286,5 \\
\hline 1993 & 4581 & 32,9 & 264,9 & 238,8 & 254,9 \\
\hline 1995 & 4453 & 32,9 & 313,4 & 227,7 & 227,7 \\
\hline 1996 & 4201 & 33,7 & 333,6 & 243,1 & 226,8 \\
\hline 1997 & 4147 & 34,1 & 324,6 & 233,0 & 233,0 \\
\hline 1998 & 3909 & 34,1 & 326,0 & 244,8 & 244,8 \\
\hline 1999 & 4166 & 34,2 & 324,1 & 247,7 & 240,7 \\
\hline 2001 & 4008 & 34,4 & 313,9 & 277,4 & 277,4 \\
\hline 2002 & 4166 & 34,4 & 313,4 & 280,4 & 280,4 \\
\hline 2003 & 4248 & 34,3 & 306,1 & 288,0 & 288,0 \\
\hline 2004 & 4432 & 34,4 & 331,6 & 294,8 & 294,8 \\
\hline 2005 & 4468 & 34,4 & 351,9 & 323,3 & 323,3 \\
\hline 2006 & 4305 & 34,7 & 375,5 & 367,0 & 367,0 \\
\hline 2007 & 4267 & 35,2 & 410,4 & 380,0 & 380,0 \\
\hline
\end{tabular}

A Tabela 3 mostra a variação de três medidas de desigualdade da distribuição da renda. Para as três é observada redução entre 1992 e 2007. Destacando o período 19952007, sem a instabilidade inflacionária dos anos anteriores, o quadro geral é de estabilidade no nível de desigualdade da distribuição do rendimento do trabalho principal dos empregados agrícolas. Regressões do índice de Gini e de $T$ contra o tempo produzem coeficientes angulares negativos, mas que não diferem estatisticamente de zero. A regressão de $L$ contra o tempo produz um coeficiente angular positivo com probabilidade caudal do teste da hipótese de nulidade igual a 2,2\%. Mas, o crescimento sistemático do valor do $L$ é observado apenas no período 1996-2001.

(5) A importância do salário mínimo como condicionante dos salários dos empregados agrícolas é analisada por Hoffmann e Oliveira (2008) e Oliveira (2009). 
Rodolfo Hoffmann

Tabela 3

Medidas de desigualdade e de polarização da distribuição do rendimento do trabalho principal dos empregados na agricultura. Brasil, 1992-2007

\begin{tabular}{l|c|c|c|c|c}
\hline \multirow{2}{*}{ Ano } & \multicolumn{3}{|c|}{ Medidas de desigualdade } & \multicolumn{2}{c}{ Medidas de polarização } \\
\cline { 2 - 6 } & Gini & $T$ & $L$ & $P_{W}$ & $P_{M}$ \\
\hline 1992 & 0,3981 & 0,3001 & 0,2950 & 0,3541 & 0,3061 \\
\hline 1993 & 0,4077 & 0,3149 & 0,3130 & 0,2982 & 0,2979 \\
\hline 1995 & 0,3535 & 0,2486 & 0,2218 & 0,2866 & 0,2978 \\
\hline 1996 & 0,3562 & 0,2482 & 0,2210 & 0,2979 & 0,3096 \\
\hline 1997 & 0,3543 & 0,2467 & 0,2235 & 0,2938 & 0,3056 \\
\hline 1998 & 0,3501 & 0,2437 & 0,2235 & 0,2874 & 0,2964 \\
\hline 1999 & 0,3535 & 0,2726 & 0,2280 & 0,2873 & 0,2934 \\
\hline 2001 & 0,3598 & 0,2503 & 0,2426 & 0,2469 & 0,2760 \\
\hline 2002 & 0,3537 & 0,2517 & 0,2381 & 0,2276 & 0,2659 \\
\hline 2003 & 0,3388 & 0,2141 & 0,2196 & 0,2236 & 0,2449 \\
\hline 2004 & 0,3452 & 0,2314 & 0,2275 & 0,2273 & 0,2572 \\
\hline 2005 & 0,3540 & 0,2532 & 0,2433 & 0,2330 & 0,2619 \\
\hline 2006 & 0,3464 & 0,2267 & 0,2351 & 0,2294 & 0,2380 \\
\hline 2007 & 0,3521 & 0,2422 & 0,2392 & 0,2311 & 0,2524 \\
\hline
\end{tabular}

A estabilidade da desigualdade da distribuição do rendimento dos empregados agrícolas é um resultado que se destaca dentro do conjunto de pesquisas que, com base nos dados da PNAD, mostram a redução da desigualdade do rendimento por pessoa economicamente ativa desde 1995 e a queda mais acentuada da desigualdade da distribuição da renda domiciliar per capita a partir de 2001 [Ver, por exemplo, Barros, Foguel e Ulyssea (2006 e 2007) e Hoffmann e Ney (2008)].

A Tabela 3 mostra, também, a variação das medidas de polarização $P_{W}$ e $P_{M}$. É notória a diminuição sistemática dos valores de 1996 a 2003. Regressões de $P_{W}$ e $P_{M}$ contra o tempo no período 1995-2007 produzem estimativas do coeficiente angular negativas e estatisticamente significativas (probabilidade caudal inferior a 0,01\%). Também foram analisadas medidas de polarização baseadas nos índices $T$ e $L$ de Theil e a conclusão é a mesma: houve redução da polarização da distribuição do rendimento do trabalho principal dos empregados agrícolas.

\section{Desigualdade entre empregados permanentes e empregados temporários e entre regiões}

Outro tema abordado por Balsadi e Graziano da Silva (2008) é o contraste entre a qualidade do emprego dos trabalhadores permanentes e dos trabalhadores temporários, com características piores para esses últimos. 
Na primeira coluna do corpo da Tabela 4 está a porcentagem de empregados permanentes na população analisada. Essa porcentagem se mantém ao redor de $55 \%$ de 1992 a 1999, cai para 47,1\% em 2003 e posteriormente aumenta um pouco, atingindo $50,6 \%$ em 2007.

As duas colunas seguintes da Tabela 4 mostram que a porcentagem de empregados com carteira de trabalho assinada tende a crescer, tanto entre os trabalhadores permanentes como entre os temporários. A razão entre essas porcentagens permite avaliar o contraste entre permanentes e temporários no que se refere à formalização do vínculo empregatício. Essa razão oscila bastante, mas é possível perceber que ela apresenta uma tendência decrescente a partir de 1998.

Tabela 4

Empregados agrícolas no Brasil: porcentagem de empregados permanentes, porcentagem com carteira de trabalho assinada, razão entre rendimentos médios de empregados permanentes e temporários e razão entre rendimentos médios dos com carteira e dos sem carteira

\begin{tabular}{c|c|c|c|c|c|c}
\hline \multirow{2}{*}{ Ano } & \multirow{2}{*}{$\begin{array}{c}\text { \% de } \\
\text { perma- } \\
\text { nentes }\end{array}$} & \multicolumn{2}{|c|}{$\begin{array}{c}\text { \% com carteira assinada } \\
\text { entre }\end{array}$} & \multirow{2}{*}{$\begin{array}{c}\text { Razão } \\
(a)\end{array}$} & \multicolumn{2}{|c}{$\begin{array}{c}\text { Razão entre rendimentos } \\
\text { médios }\end{array}$} \\
& nentes $(a)$ & $\begin{array}{c}\text { Tempo- } \\
\text { rários }(b)\end{array}$ & $\frac{2}{(b)}$ & $\begin{array}{c}\text { perm./ } \\
\text { temp. }\end{array}$ & $\begin{array}{c}\text { com cart./ } \\
\text { sem cart. }\end{array}$ \\
\hline 1992 & 54,5 & 37,8 & 7,8 & 4,85 & 1,63 & 2,02 \\
\hline 1993 & 55,8 & 39,7 & 7,6 & 5,23 & 1,71 & 2,04 \\
\hline 1995 & 56,1 & 40,5 & 9,0 & 4,49 & 1,46 & 1,65 \\
\hline 1996 & 57,8 & 41,1 & 12,6 & 3,25 & 1,37 & 1,57 \\
\hline 1997 & 55,6 & 42,8 & 9,8 & 4,34 & 1,58 & 1,73 \\
\hline 1998 & 55,3 & 44,1 & 6,7 & 6,62 & 1,63 & 1,75 \\
\hline 1999 & 55,6 & 45,2 & 9,3 & 4,85 & 1,61 & 1,66 \\
\hline 2001 & 52,4 & 45,7 & 8,1 & 5,66 & 1,76 & 1,83 \\
\hline 2002 & 50,5 & 45,4 & 11,7 & 3,89 & 1,64 & 1,84 \\
\hline 2003 & 47,1 & 46,8 & 13,4 & 3,48 & 1,60 & 1,73 \\
\hline 2004 & 47,1 & 50,6 & 14,5 & 3,50 & 1,64 & 1,85 \\
\hline 2005 & 48,3 & 51,3 & 13,4 & 3,83 & 1,68 & 1,92 \\
\hline 2006 & 48,2 & 51,0 & 16,6 & 3,07 & 1,62 & 2,01 \\
\hline 2007 & 50,6 & 52,5 & 17,5 & 2,99 & 1,68 & 1,92 \\
\hline
\end{tabular}

Outra maneira de comparar a qualidade do emprego de trabalhadores permanentes e temporários é a razão entre os respectivos rendimentos médios. Excetuando-se os valores mais baixos em 1995 e 1996, essa razão se mantém quase sempre entre 1,6 e 1,7. Essa relação entre rendimentos de empregados permanentes e temporários certamente subestima a diferença na qualidade do emprego, pois para um empregado temporário o rendimento no mês de referência da PNAD pode superestimar seu rendimento médio ao longo do ano, se ele fica um período desempregado.

A última coluna da Tabela 4 mostra que o contraste entre rendimentos médios é mais intenso quando se comparam empregados com carteira e empregados sem carteira, com os com carteira chegando a ganhar, em média, mais do que o dobro da remuneração dos sem carteira. No período 1995-2007 a razão entre rendimentos médios dos empregados agrícolas com carteira e sem carteira apresenta tendência crescente. Pode-se dizer que no período 1995-2007 houve, na agricultura brasileira, um processo de polarização entre os empregados com carteira e os sem carteira, no que se refere ao valor da sua remuneração. 
Uma possível explicação para isso é que os salários dos empregados com carteira foram mais diretamente beneficiados pelo crescimento do valor real do salário mínimo no período 1995-2007 (ver Oliveira, 2008, especialmente a seção 5.5.2).

A Tabela 5 permite analisar a evolução da importância da diferença entre trabalhadores permanentes e temporários na desigualdade da distribuição do rendimento dos empregados agrícolas no Brasil. Cabe ressaltar que a desigualdade entre duas categorias não depende apenas da razão entre os rendimentos médios, mas também da proporção da população que pertence a cada categoria.

Tabela 5

Desigualdade entre empregados temporários e permanentes na distribuição do rendimento do trabalho principal dos empregados agrícolas no Brasil, 1992-2007.

Medida da desigualdade entre as duas categorias e sua participação na desigualdade total

\begin{tabular}{r|c|c|c|r|r|r}
\hline \multirow{2}{*}{ Ano } & \multicolumn{2}{|c|}{ Índice de Gini } & \multicolumn{2}{c|}{$T$ de Theil } & \multicolumn{2}{c}{$L$ de Theil } \\
\cline { 2 - 7 } & $\begin{array}{c}\text { entre } \\
\text { categorias }\end{array}$ & \% do total & $\begin{array}{c}\text { entre } \\
\text { categorias }\end{array}$ & \% do total & $\begin{array}{c}\text { entre } \\
\text { categorias }\end{array}$ & $\%$ do total \\
\hline 1992 & 0,1167 & 29,3 & 0,0281 & 9,4 & 0,0291 & 9,9 \\
\hline 1993 & 0,1261 & 30,9 & 0,0332 & 10,6 & 0,0347 & 11,1 \\
\hline 1995 & 0,0895 & 25,3 & 0,0166 & 6,7 & 0,0171 & 7,7 \\
\hline 1996 & 0,0737 & 20,7 & 0,0114 & 4,6 & 0,0116 & 5,3 \\
\hline 1997 & 0,1081 & 30,5 & 0,0243 & 9,8 & 0,0251 & 11,2 \\
\hline 1998 & 0,1160 & 33,1 & 0,0280 & 11,5 & 0,0290 & 13,0 \\
\hline 1999 & 0,1127 & 31,9 & 0,0264 & 9,7 & 0,0274 & 12,0 \\
\hline 2001 & 0,1350 & 37,5 & 0,0373 & 14,9 & 0,0387 & 15,9 \\
\hline 2002 & 0,1212 & 34,3 & 0,0297 & 11,8 & 0,0304 & 12,8 \\
\hline 2003 & 0,1172 & 34,6 & 0,0276 & 12,9 & 0,0278 & 12,7 \\
\hline 2004 & 0,1222 & 35,4 & 0,0300 & 13,0 & 0,0303 & 13,3 \\
\hline 2005 & 0,1281 & 36,2 & 0,0330 & 13,1 & 0,0336 & 13,8 \\
\hline 2006 & 0,1190 & 34,3 & 0,0284 & 12,5 & 0,0288 & 12,3 \\
\hline 2007 & 0,1265 & 35,9 & 0,0324 & 13,4 & 0,0332 & 13,9 \\
\hline
\end{tabular}

Observa-se que a desigualdade entre as duas categorias representa uma porcentagem muito maior da desigualdade total para o índice de Gini do que para as medidas de Theil. Isso está associado ao fato de o índice de Gini não ser uma medida de desigualdade aditivamente decomponível, como já foi assinalado no final da seção 1. Para as medidas de Theil, a medida da desigualdade total é igual à soma da medida da desigualdade entre categorias com uma média ponderada das medidas da desigualdade dentro das categorias, mas isso não é verdade para o índice de Gini.

Verifica-se, na Tabela 5, que para as três medidas de desigualdade utilizadas, a desigualdade entre empregados permanentes e temporários diminui de 1993 a 1996, em seguida aumenta até 2001 e depois permanece em patamar relativamente elevado até 2007. O comportamento é muito semelhante para a participação porcentual da desigualdade entre as duas categorias na desigualdade global.

A Tabela 6 mostra o mesmo tipo de análise, considerando a diferença entre empregados com carteira e empregados sem carteira de trabalho assinada. Observa-se que a desigualdade entre essas categorias é relativamente mais importante do que a 
desigualdade entre empregados permanentes e temporários, chegando a representar mais de $20 \%$ da desigualdade total para as medidas aditivamente decomponíveis ( $T$ e $L$ de Theil).

Tabela 6

Desigualdade entre empregados com e sem carteira de trabalho assinada na distribuição do rendimento do trabalho principal dos empregados agrícolas no Brasil, 1992-2007. Medida da desigualdade entre as duas categorias e sua participação na desigualdade total

\begin{tabular}{c|c|c|c|c|c|c}
\hline \multirow{2}{*}{ Ano } & \multicolumn{2}{|c|}{ Índice de Gini } & \multicolumn{2}{c|}{$T$ de Theil } & \multicolumn{2}{c}{$L$ de Theil } \\
\cline { 2 - 7 } & $\begin{array}{c}\text { entre } \\
\text { categorias }\end{array}$ & \% do total & $\begin{array}{c}\text { entre } \\
\text { categorias }\end{array}$ & \% do total & $\begin{array}{c}\text { entre } \\
\text { categorias }\end{array}$ & $\%$ do total \\
\hline 1992 & 0,1501 & 37,7 & 0,0551 & 18,4 & 0,0505 & 17,1 \\
\hline 1993 & 0,1557 & 38,2 & 0,0576 & 18,3 & 0,0531 & 17,0 \\
\hline 1995 & 0,1081 & 30,6 & 0,0278 & 11,2 & 0,0262 & 11,8 \\
\hline 1996 & 0,1010 & 28,4 & 0,0234 & 9,4 & 0,0223 & 10,1 \\
\hline 1997 & 0,1222 & 34,5 & 0,0344 & 13,9 & 0,0324 & 14,5 \\
\hline 1998 & 0,1238 & 35,4 & 0,0357 & 14,7 & 0,0336 & 15,0 \\
\hline 1999 & 0,1142 & 32,3 & 0,0296 & 10,9 & 0,0281 & 12,3 \\
\hline 2001 & 0,1357 & 37,7 & 0,0424 & 16,9 & 0,0398 & 16,4 \\
\hline 2002 & 0,1386 & 39,2 & 0,0435 & 17,3 & 0,0410 & 17,2 \\
\hline 2003 & 0,1238 & 36,5 & 0,0347 & 16,2 & 0,0329 & 15,0 \\
\hline 2004 & 0,1451 & 42,0 & 0,0458 & 19,8 & 0,0437 & 19,2 \\
\hline 2005 & 0,1547 & 43,7 & 0,0518 & 20,5 & 0,0494 & 20,3 \\
\hline 2006 & 0,1681 & 48,5 & 0,0600 & 26,5 & 0,0577 & 24,5 \\
\hline 2007 & 0,1585 & 45,0 & 0,0525 & 21,7 & 0,0509 & 21,3 \\
\hline
\end{tabular}

Verifica-se que as medidas de desigualdade entre empregados com carteira e empregados sem carteira, assim como as respectivas participações na desigualdade da distribuição do rendimento dos empregados agrícolas, apresentam tendência crescente no período 1995-2007. Isso está associado ao crescimento da razão entre os rendimentos médios das duas categorias, já analisado anteriormente.

Como não foi constatada tendência de crescimento da desigualdade da distribuição do rendimento de todos os empregados agrícolas, o crescimento da desigualdade entre os com carteira e os sem carteira deve ter sido compensado por redução em outra parcela da desigualdade. Pode-se verificar que, no período 1995-2007 a desigualdade dentro do grupo dos sem carteira é estável, mas a desigualdade dentro do grupo dos com carteira mostra tendência decrescente, com o índice de Gini diminuindo de 0,3107 em 1995 para 0,2417 em 2007.

O contraste no comportamento da desigualdade é maior quando se comparam os empregados temporários com os permanentes. No período 1997-2007, a desigualdade cresce dentro do grupo dos temporários, mas diminui dentro do grupo dos permanentes.

É interessante analisar o diferente papel do salário mínimo na distribuição do rendimento do trabalho principal dessas categorias de empregados. Para os empregados permanentes, desde 1995 o primeiro quartil é igual ao salário mínimo. Para os empregados com carteira, em todo o período 1992-2007 o primeiro quartil é pouco superior ou igual ao salário mínimo. Para os empregados temporários, o salário mínimo é igual à mediana em 1995 e 1996 e a partir de 2001 é igual ou um pouco inferior ao terceiro quartil. Para os 
empregados sem carteira, o salário mínimo é igual à mediana no período 1995-1998 e é igual ao terceiro quartil no período 2002-2007. Para os empregados temporários ou sem carteira de trabalho, o salário mínimo, a partir de 2001, tem funcionado como balizador dos rendimentos relativamente elevados (terceiro quartil) dessas categorias. É possível que o intenso crescimento do valor real do salário mínimo no período 1995-2007, que foi uma das causas da redução da desigualdade da distribuição da renda no Brasil, tenha contribuído para aumentar a desigualdade dentro da categoria dos empregados agrícolas temporários.

Outro aspecto abordado por Balsadi e Graziano da Silva (2008) é o contraste entre diversas categorias de empregados no que se refere ao nível de escolaridade. A Tabela 7 mostra a escolaridade média dos empregados agrícolas permanentes e temporários no período 1992-2007 e, na última coluna, a relação entre as escolaridades médias das duas categorias. Os níveis médios de escolaridade são notoriamente baixos e a tendência de crescimento pode ser considerada muito lenta. A relação entre escolaridades médias de empregados permanentes e temporários apresenta tendência decrescente a partir de 1998, indicando que ocorre redução das diferenças relativas no nível de escolaridade.

Tabela 7

Escolaridade média dos empregados agrícolas, dos empregados permanentes e dos empregados temporários. Brasil, 1992-2007

\begin{tabular}{c|c|c|c|c}
\hline \multirow{2}{*}{ Ano } & \multicolumn{2}{|c|}{ Escolaridade média dos empregados agrícolas } & \multirow{2}{*}{$\begin{array}{c}\text { Razão } \\
(a)\end{array}$} \\
\cline { 3 - 4 } & todos & $\begin{array}{c}\text { permanentes } \\
(a)\end{array}$ & $\begin{array}{c}\text { temporários } \\
(b)\end{array}$ & $(b)$ \\
\hline 1992 & 2,09 & 2,32 & 1,81 & 1,28 \\
\hline 1993 & 2,25 & 2,52 & 1,90 & 1,32 \\
\hline 1995 & 2,25 & 2,48 & 1,95 & 1,28 \\
\hline 1996 & 2,43 & 2,67 & 2,09 & 1,28 \\
\hline 1997 & 2,43 & 2,73 & 2,05 & 1,33 \\
\hline 1998 & 2,60 & 2,93 & 2,19 & 1,34 \\
\hline 1999 & 2,74 & 3,06 & 2,33 & 1,31 \\
\hline 2001 & 2,79 & 3,11 & 2,43 & 1,28 \\
\hline 2002 & 2,92 & 3,20 & 2,64 & 1,21 \\
\hline 2003 & 3,16 & 3,48 & 2,88 & 1,21 \\
\hline 2004 & 3,40 & 3,66 & 3,16 & 1,16 \\
\hline 2005 & 3,47 & 3,80 & 3,16 & 1,20 \\
\hline 2006 & 3,64 & 3,95 & 3,35 & 1,18 \\
\hline 2007 & 3,84 & 4,17 & 3,50 & 1,19 \\
\hline
\end{tabular}

Para finalizar, a Tabela 8 mostra como se comportou a desigualdade entre 6 regiões do país: Norte (urbano), Nordeste, o conjunto dos estados de Minas Gerais, Espírito Santo e Rio de Janeiro, o estado de São Paulo, Sul e Centro-Oeste. As regressões das três medidas de desigualdade contra o tempo no período 1995-2007 produzem coeficientes angulares positivos e com probabilidade caudal do teste de nulidade entre $6 \%$ e $10 \%$. Quando se considera como variável dependente a participação porcentual da desigualdade inter-regional na desigualdade total, no caso do índice de Gini e da medida $T$ constata-se que há tendência de crescimento (probabilidade caudal abaixo de $2 \%$ ). Há, portanto, crescimento da importância relativa da desigualdade inter-regional depois de 1995. 
Tabela 8

Desigualdade entre 6 regiões (Norte, Nordeste, MG + ES + RJ, SP, Sul e Centro-Oeste) na distribuição do rendimento do trabalho principal dos empregados agrícolas no Brasil, 1992-2007.

Medida da desigualdade entre regiões e sua participação na desigualdade total

\begin{tabular}{c|c|c|c|c|c|c}
\hline \multirow{2}{*}{ Ano } & \multicolumn{2}{|c|}{ Índice de Gini } & \multicolumn{2}{c|}{$T$ de Theil } & \multicolumn{2}{c}{$L$ de Theil } \\
\cline { 2 - 7 } & $\begin{array}{c}\text { entre } \\
\text { regiões }\end{array}$ & $\%$ do total & $\begin{array}{c}\text { entre } \\
\text { regiões }\end{array}$ & \% do total & $\begin{array}{c}\text { entre } \\
\text { regiões }\end{array}$ & $\%$ do total \\
\hline 1992 & 0,1808 & 45,4 & 0,0528 & 17,6 & 0,0537 & 18,2 \\
\hline 1993 & 0,1973 & 48,4 & 0,0639 & 20,3 & 0,0657 & 21,0 \\
\hline 1995 & 0,1593 & 45,1 & 0,0409 & 16,4 & 0,0412 & 18,6 \\
\hline 1996 & 0,1749 & 49,1 & 0,0501 & 20,2 & 0,0503 & 22,7 \\
\hline 1997 & 0,1703 & 48,1 & 0,0467 & 18,9 & 0,0466 & 20,8 \\
\hline 1998 & 0,1698 & 48,5 & 0,0469 & 19,3 & 0,0475 & 21,2 \\
\hline 1999 & 0,1774 & 50,2 & 0,0510 & 18,7 & 0,0510 & 22,4 \\
\hline 2001 & 0,1728 & 48,0 & 0,0490 & 19,6 & 0,0488 & 20,1 \\
\hline 2002 & 0,1776 & 50,2 & 0,0514 & 20,4 & 0,0518 & 21,8 \\
\hline 2003 & 0,1633 & 48,2 & 0,0437 & 20,4 & 0,0436 & 19,9 \\
\hline 2004 & 0,1668 & 48,3 & 0,0450 & 19,4 & 0,0452 & 19,9 \\
\hline 2005 & 0,1868 & 52,8 & 0,0578 & 22,8 & 0,0571 & 23,5 \\
\hline 2006 & 0,1820 & 52,5 & 0,0545 & 24,1 & 0,0553 & 23,5 \\
\hline 2007 & 0,1773 & 50,3 & 0,0514 & 21,2 & 0,0525 & 21,9 \\
\hline
\end{tabular}

\section{Conclusões}

Os dados da PNAD mostram substancial diminuição da desigualdade da distribuição da renda entre pessoas ocupadas (ou entre pessoas economicamente ativas) no Brasil, no período 1995-2007. Entretanto, quando se analisa a distribuição do rendimento do trabalho principal dos empregados no setor agrícola, constata-se que o grau de desigualdade permanece estável nesse período. Verifica-se, também, que houve redução no grau de polarização dessa distribuição.

A remuneração média dos empregados permanentes permanece cerca de $70 \%$ acima da remuneração dos empregados temporários. A diferença relativa é maior quando se comparam os rendimentos médios de empregados com ou sem carteira de trabalho assinada. Verifica-se que a desigualdade entre essas duas categorias apresenta tendência de crescimento no período 1995-2007, levando a quase dobrar sua participação na desigualdade total da distribuição do rendimento do trabalho principal dos empregados agrícolas.

A desigualdade dentro do grupo de empregados permanentes ou dentro do grupo dos com carteira apresenta tendência decrescente, mas o mesmo não acontece para os empregados temporários ou para os sem carteira. Pelo menos em parte, isso se deve ao papel do salário mínimo, cujo valor real cresceu substancialmente a partir de 1996. Para os empregados permanentes e para os com carteira o salário mínimo é um balizador das remunerações mais baixas, mas para os empregados temporários e os sem carteira, pelo menos a partir de 2002, o salário mínimo passa a balizar os rendimentos relativamente elevados dessas categorias (coincidindo, quase sempre, com o terceiro quartil da distribuição dos rendimentos da categoria). 
Rodolfo Hoffmann

A proporção de empregados com carteira, que é um indicador básico da formalização dos vínculos empregatícios, mostra clara tendência crescente, tanto entre empregados permanentes como entre empregados temporários.

\section{Referências bibliográficas}

BARROS, R. P.; FOGUEL, M. N.; ULYSSEA, G. (Org.). Desigualdade de renda no Brasil: uma análise da queda recente. Brasília: Ipea, 2006 e 2007. v. 1-2.

BALSADI, O. V. O mercado de trabalho assalariado na agricultura brasileira e suas diferenciações regionais no período 1992-204. São Paulo: Aderaldo e Rothschild (HUCITEC): Ordem dos Economistas do Brasil, 2008.

; GRAZIANO DA SILVA, J. A polarização da qualidade do emprego na agricultura brasileira no período 1992-2004. Economia e Sociedade, Campinas, v. 17, n. 3 (34), p. 343-524, dez. 2008.

ESTEBAN, J.; RAY, D. On the measurement of polarization. Econometrica, v. 62, n. 4, p. 819-851, 1994.

HOFFMANN, R. Distribuição de renda: medidas de desigualdade e pobreza. São Paulo: Editora da Universidade de São Paulo, 1998.

Polarização da distribuição de renda no Brasil. Econômica, Rio de Janeiro, v. 10, n. 2, dez. 2008 (no prelo).

; NEY, M. G. A recente queda da desigualdade de renda no Brasil: análise de dados da PNAD, do Censo Demográfico e das Contas Nacionais. Econômica, Rio de Janeiro, v. 10, n. 1, p. 7-39, jun. 2008.

; OLIVEIRA, F. C. R. Evolução da remuneração das pessoas empregadas na cana-de-açúcar e em outras lavouras, no Brasil e em São Paulo. In: CONGRESSO DA SOCIEDADE BRASILEIRA DE ECONOMIA, ADMINISTRAÇÃO E SOCIOLOGIA RURAL, 46, Rio Branco, 20-23 jul. 2008. Anais em CD-ROM... Rio Branco: Sober, 2008.

OLIVEIRA, F. C. R. Ocupação, emprego e remuneração na cana-de-açúcar e em outras atividades agropecuárias no Brasil, de 1992 a 2007. Dissertação (Mestrado em Economia Aplicada)- ESALQ-USP, Piracicaba, 2008. Disponível em: www.esalq.usp.br/gemt.

WOLFSON, M. C. When inequalities diverge. American Economic Review, v. 84, n. 2, p. 353-358, 1994. 